\title{
2020 Reviewer Acknowledgement
}

\author{
Editorial Office of Journal of Clinical and Translational Hepatology
}

We thank the following reviewers for their contribution and support in 2020.

\begin{tabular}{|c|c|c|}
\hline $\begin{array}{l}\text { MaiAli Abd El Meguid } \\
\text { EGYPT }\end{array}$ & $\begin{array}{l}\text { Harjot Bedi } \\
\text { CANADA }\end{array}$ & $\begin{array}{l}\text { Watcharasak Chotiyaputta } \\
\text { THAILAND }\end{array}$ \\
\hline $\begin{array}{l}\text { Sherief Abd-Elsalam } \\
\text { EGYPT }\end{array}$ & $\begin{array}{l}\text { Manas Kumar Behera } \\
\text { INDIA }\end{array}$ & $\begin{array}{l}\text { Emanuela Ciracì } \\
\text { ITALY }\end{array}$ \\
\hline $\begin{array}{l}\text { Anant Agarwalla } \\
\text { UNITED STATES }\end{array}$ & $\begin{array}{l}\text { Rahima Bhanji } \\
\text { CANADA }\end{array}$ & $\begin{array}{l}\text { Andrea Dalbeni } \\
\text { ITALY }\end{array}$ \\
\hline $\begin{array}{l}\text { Muhammad Ali Ahad } \\
\text { SAUDI ARABIA }\end{array}$ & $\begin{array}{l}\text { Ines Bilic-Curcic } \\
\text { CROATIA }\end{array}$ & $\begin{array}{l}\text { Babak Daneshfard } \\
\text { IRAN }\end{array}$ \\
\hline $\begin{array}{l}\text { Nobuhisa Akamatsu } \\
\text { JAPAN }\end{array}$ & $\begin{array}{l}\text { John w } \mathbf{~ B i r k} \\
\text { UNITED STATES }\end{array}$ & $\begin{array}{l}\text { Mohamed A Daw } \\
\text { LIBYAN ARAB JAMAHIRIYA }\end{array}$ \\
\hline $\begin{array}{l}\text { Kawtar Alkhalloufi } \\
\text { UNITED STATES }\end{array}$ & $\begin{array}{l}\text { Kyle E Brown } \\
\text { UNITED STATES }\end{array}$ & $\begin{array}{l}\text { C Dimacali } \\
\text { SINGAPORE }\end{array}$ \\
\hline $\begin{array}{l}\text { Gianfranco D Alpini } \\
\text { UNITED STATES }\end{array}$ & $\begin{array}{l}\text { Peter Buch } \\
\text { UNITED STATES }\end{array}$ & $\begin{array}{l}\text { Jianqiang Ding } \\
\text { CHINA }\end{array}$ \\
\hline $\begin{array}{l}\text { Khaled Amer } \\
\text { EGYPT }\end{array}$ & $\begin{array}{l}\text { Chalermrat Bunchorntavakul } \\
\text { THAILAND }\end{array}$ & $\begin{array}{l}\text { Xiaoguang Dou } \\
\text { CHINA }\end{array}$ \\
\hline $\begin{array}{l}\text { Masahiro Arai } \\
\text { JAPAN }\end{array}$ & $\begin{array}{l}\text { Wenqing (Wendy) Cao } \\
\text { UNITED STATES }\end{array}$ & $\begin{array}{l}\text { Iliana B Doycheva } \\
\text { UNITED STATES }\end{array}$ \\
\hline $\begin{array}{l}\text { Teruko Arinaga-Hino } \\
\text { JAPAN }\end{array}$ & $\begin{array}{l}\text { Guido Carpino } \\
\text { ITALY }\end{array}$ & $\begin{array}{l}\text { Atanu Kumar Dutta } \\
\text { INDIA }\end{array}$ \\
\hline $\begin{array}{l}\text { Tarik Asselah } \\
\text { FRANCE }\end{array}$ & $\begin{array}{l}\text { Ryan Chadha } \\
\text { UNITED STATES }\end{array}$ & $\begin{array}{l}\text { Mohamed A. El-Guindi } \\
\text { EGYPT }\end{array}$ \\
\hline $\begin{array}{l}\text { Hamed Azimi } \\
\text { UNITED STATES }\end{array}$ & $\begin{array}{l}\text { Phunchai Charatcharoenwitthaya } \\
\text { THAILAND }\end{array}$ & $\begin{array}{l}\text { Yu-Chen Fan } \\
\text { CHINA }\end{array}$ \\
\hline $\begin{array}{l}\text { Olufemi Emmanuel Babalola } \\
\text { NIGERIA }\end{array}$ & $\begin{array}{l}\text { Po-Hung Chen } \\
\text { UNITED STATES }\end{array}$ & $\begin{array}{l}\text { Christopher Fan } \\
\text { UNITED STATES }\end{array}$ \\
\hline $\begin{array}{l}\text { Gyorgy Baffy } \\
\text { UNITED STATES }\end{array}$ & $\begin{array}{l}\text { Yongpeng Chen } \\
\text { CHINA }\end{array}$ & $\begin{array}{l}\text { Piera Federico } \\
\text { ITALY }\end{array}$ \\
\hline $\begin{array}{l}\text { Shashwatee Bagchi } \\
\text { UNITED STATES }\end{array}$ & $\begin{array}{l}\text { En-Qiang Chen } \\
\text { CHINA }\end{array}$ & $\begin{array}{l}\text { Gong Feng } \\
\text { CHINA }\end{array}$ \\
\hline $\begin{array}{l}\text { Dibyajyoti Banerjee } \\
\text { INDIA }\end{array}$ & $\begin{array}{l}\text { Xiaoping Chen } \\
\text { CHINA }\end{array}$ & $\begin{array}{l}\text { Annarosa Floreani } \\
\text { ITALY }\end{array}$ \\
\hline $\begin{array}{l}\text { Jodie A Barkin } \\
\text { UNITED STATES }\end{array}$ & $\begin{array}{l}\text { Lixian Chen } \\
\text { UNITED STATES }\end{array}$ & $\begin{array}{l}\text { Yanhang Gao } \\
\text { CHINA }\end{array}$ \\
\hline $\begin{array}{l}\text { Giorgia Beatrice } \\
\text { ITALY }\end{array}$ & $\begin{array}{l}\text { Min Chen } \\
\text { CHINA }\end{array}$ & $\begin{array}{l}\text { Sunir J Garg } \\
\text { UNITED STATES }\end{array}$ \\
\hline${ }^{*} D O I: 10.1$ & $\begin{array}{l}\text { Aziz Alami Chentoufi } \\
\text { CANADA }\end{array}$ & $\begin{array}{l}\text { Mauro Giuffre } \\
\text { ITALY }\end{array}$ \\
\hline
\end{tabular}


Amit Goel

INDIA

George Boon-Bee Goh

SINGAPORE

Humberto C Gonzalez

UNITED STATES

Yuecheng Guo

CHINA

Jinlong Guo

SINGAPORE

Julio A Gutierrez

UNITED STATES

Tao Han

CHINA

Ying Han

CHINA

Ma Ai Thanda Han

UNITED STATES

Guo-Rong Han

CHINA

Tao Han

CHINA

Kazuhiko Hayashi

JAPAN

Marija Heffer

CROATIA

Jeong Heo

KOREA

David J. Hermel

UNITED STATES

Cheng-Maw Ho

TAIWAN

Yan Huang

CHINA

Chiung-Kuei Huang

UNITED STATES

Junyu Huo

CHINA

Tatsuo Kanda

JAPAN

Qian Kang

CHINA

Sandeep Kumar Karn

CHINA

Chandan Kumar Kedarisetty

INDIA

Lindsey Kennedy

UNITED STATES

Hyunseok Kim

UNITED STATES

Ahyoung Kim

UNITED STATES
Arno Kornberg

GERMANY

John Koskinas

GREECE

Ramesh Kudira

UNITED STATES

Tasha Kulai

CANADA

Anand V. Kulkarni

INDIA

Ravikant Kumar

INDIA

Pramod Kumar

INDIA

Karan Kumar

INDIA

Ewa Kurys-Denis

POLAND

Sundeep Lakhtakia

INDIA

Xiang-Ming Lao

CHINA

George Lau

HONG KONG

Ruoqing li

CHINA

Chen Li

GERMANY

Jing Li

CHINA

Qingjiao Liao

CHINA

Joseph K Lim

UNITED STATES

Vivek Lingiah

UNITED STATES

Shousheng Liu

CHINA

Cheng-Hai Liu

CHINA

Xiaocong Liu

UNITED STATES

Xiaolin Liu

CHINA

Man-Qing Liu

CHINA

Lungen Lu

CHINA

Sunil K Malonia UNITED STATES

Alessandro Mantovani ITALY
Vladimir Marquez

CANADA

Rosa Maria Martin-Mateos

SPAIN

Krzysztof Marycz

POLAND

Muhammad Masroor

PAKISTAN

Mitchell R McGill

UNITED STATES

Magnus McLeod

CANADA

Matthew McMillin

UNITED STATES

Ahmed Atef Mesalam

EGYPT

Katarina Miskovic Spoljaric CROATIA

Søren Møller

DENMARK

Harikumar R. Nair

UNITED KINGDOM

Seyedehsan Navabi

UNITED STATES

Paul Naylor

UNITED STATES

Bolin Niu

UNITED STATES

Jean-Baptiste Nousbaum

FRANCE

Jason Pan

UNITED STATES

Charles Panackel

INDIA

James S Park

UNITED STATES

Lei Pei

CHINA

Chuang Peng

CHINA

María Teresa Pérez-Gracia

SPAIN

Cyriac Abby Philips

INDIA

Paul J. Pockros

UNITED STATES

M. Pokorska-Śpiewak POLAND

Madhumita Premkumar INDIA

Michele Pritchard

UNITED STATES 
Kittichai Promrat

UNITED STATES

Atoosa Rabiee

UNITED STATES

Alok Ranjan

UNITED STATES

Pravin M Rathi

INDIA

\section{Aathira Ravindranath \\ INDIA}

Sammy Saab

UNITED STATES

Mehdi Sadat

IRAN

Ghada Salum

EGYPT

Filippo Sartori

ITALY

Jinendra L Satiya

UNITED STATES

Moinak Sen Sarma

INDIA

Neeral L Shah

UNITED STATES

Shalimar

INDIA

Mithun Sharma

INDIA

Vishal Sharma

INDIA

Jifang Sheng

CHINA

Hao Shi

UNITED STATES

Surajit Sinha

UNITED STATES

Coleman Smith

UNITED STATES

Martina Smolic

CROATIA

Philippe Sogni

FRANCE
Bin Song

CHINA

Vikrant Sood

INDIA

Siddharth Sridhar

CHINA

N Stefan

GERMANY

Michael Stewart

CANADA

Sridhar Sundaram

INDIA

R Tang

CHINA

Ni Tang

CHINA

Tawesak Tanwandee

THAILAND

Norberto Chavez Tapia

MEXICO

Rolf Teschke

GERMANY

Peng-sheng Ting

UNITED STATES

Parijat Ram Tripathi

INDIA

Georgios Tsoulfas

GREECE

Shunsuke Uno

JAPAN

Jaimy Villavicencio Kim

UNITED STATES

Lucija Virovic-Jukic

CROATIA

Lili Wang

UNITED STATES

Grace Lai-Hung Wong

HONG KONG

Yu Jun Wong

SINGAPORE

Benjamin I Woolbright

UNITED STATES
Chencheng Xie UNITED STATES

Hui Xie

CHINA

Yongning Xin

CHINA

Weijie Xu

CHINA

Tao Yang

CHINA

Ling Yang

CHINA

Yulong Yang

CHINA

Eric M Yoshida

CANADA

Kentaro Yoshioka

JAPAN

Yanli Zeng

CHINA

Yuanyuan Zhang

CHINA

Dazhi Zhang

CHINA

Lanjing Zhang

UNITED STATES

Hao Zhang

CHINA

Yao Zhao

CHINA

Ze-Hua Zhao

CHINA

Ming-Hua Zheng

CHINA

Jianming Zheng CHINA

Jian-Hong Zhong

CHINA

Da Zhou

CHINA

Julie Zhu

CANADA 